\title{
Can Botulinum toxin type A prevent kinetic horizontal forehead lines to become static? A five year follow up study
}

\author{
Ahmed F ElSherief, MD; Sameh M El-Taher, MD; Asser El-Hilaly, MD \\ Plastic and Reconstructive Surgery Department, Ain Shams University, \\ Cairo, Egypt.
}

\begin{abstract}
Background: Botulinum toxin type A injection has become a worldwide leading method for treating facial rhytides specially in women. Authors still debate whether or not regular administration of the toxin may delay or prevent kinetic horizontal forehead lines from changing their nature to static ones and hence the need for further interventions including facial cosmetic surgery. This study was performed to analyze the relationship between regular administrations of Botulinum toxin type A injection and the change in nature of horizontal forehead lines.

Methods: Forty female patients, whose ages ranged from 35 to 45 years, were divided into two groups of twenty each. Group A included females who had regular administration (2-3 times annually) of Botulinum toxin type A to treat horizontal forehead lines. Group B included twenty females who infrequently (once every 12-18 months) had Botulinum toxin type A injection. Changes in nature of the horizontal forehead lines were compared between the two groups, according to the Carruther's five grade scale for forehead wrinkles established in 2008.

Results: Eighty five percent of Group A patients who had regular Botulinum toxin type A injections, showed no change to their forehead lines after five years, and only $15 \%$ of the group showed one grade change. On the contrary, five years later, $80 \%$ of group B females showed change in the nature of their forehead wrinkles one or two grades more, according to the Carruther's scale of forehead rhytides.

Conclusion: Regular administration of Botulinum toxin type A during its effective period of action (4 to 6 months), two or three times per year, prevents progression of forehead horizontal wrinkles from changing their kinetic nature over a period of five years.
\end{abstract}

\section{Introduction:}

Although the non-cosmetic effect of Botulinum toxin type A (Botox) was first noticed in 1822, the "magic of Botox" was only widely appreciated around the world in 2002, when FDA approval of its cosmetic usage was announced. This was achieved through the effort of the "Carruthers" of Vancouver Canada, and hence the toxin's widespread recognition. ${ }^{1}$

Over the years and due to cumulative effect of both internal factors (aging and tissue wear and tear) and external environmental factors (sun exposure and smoking), the skin tends to lose its elasticity and shows signs of aging. ${ }^{2}$ Contractions of the facial muscles results in different patterns of facial expressions. The long term contraction of muscles gradually produces its toll on the skin, with the appearance of lines or rhytides. As individuals age, compensatory increase of the visual field is aided by the action of frontalis muscle which is commonly used to exhaustion. ${ }^{3}$

Non-surgical enhancement of facial rhytides using Botulinum toxin type A injection has been the choice of many patients over surgery for the past years. In addition, other complementary methods as hyaluronic acid, chemical peeling or laser resurfacing much enhance the effect of injections. These procedures do not completely prevent surgery 
in the future, but rather delay the need for surgery at a younger age. ${ }^{4}$

The mode of function of Botulinum toxin type $\mathrm{A}$ is to block acetyl choline release from cholinergic motor nerve endings from motor end plate receptors. Muscle motion is thus affected, and according to the amount of toxin units injected per area, muscles can vary from moderate to minimal motion, to becoming temporarily flaccid. The effect of the Botulinum toxin ranges from 4 to 6 months then is completely reversible. During the effective period of action, muscle mobility is less, and thus muscle pull on the skin is also decreased. This in turn helps to render the expression lines less affected. The overall appearance is a face with little or no wrinkles. Basic units of Botulinum toxin type A applied to the forehead range from 15 to 25 units, according to the patient's age, depth of rhytides and number of forehead lines apparent. $^{5}$

With advancement of age, deep folds become more obvious, and redundant lax skin could better be enhanced only through surgical procedures. The regular treatment by non-surgical procedures thus delays the need for surgery, and together with a healthy life style and healthy diet, may help avoiding surgery permanently according to individual satisfaction with their results. ${ }^{6}$

Glogau in 1996 classified forehead lines into four grades, and the Carruthers in 2008 further modified the classification into five. ${ }^{7}$ Grade I no wrinkles, grade II kinetic wrinkles, grade III wrinkles at rest that become deeper by frontalis muscle contraction (static-kinetic), grade IV wrinkles that show no difference in appearance at rest or during muscle action "completely static", or static wrinkles that disappear by skin stretch, and grade $\mathrm{V}$ forehead wrinkles that don't improve by skin stretch, or more than two lines of forehead wrinkles. The classification was based on the main factors affecting facial wrinkles, mainly age, smoking, and sun exposure. ${ }^{8}$

Aim of the work: This study was performed to analyze the relationship between regular administrations of Botulinum toxin type A injection 2-3 times per year and the change in nature of horizontal forehead lines.

\section{Patients and methods:}

This study included a total of forty women, ranging from 35 to 45 years old. They were divided into two groups $\mathrm{A} \& \mathrm{~B}$, with a total of twenty women in each group. Group A patients included females who had Botulinum toxin $\mathrm{A}$ injected at regular time intervals of 4 to 6 months apart, while Group B included patients who had the toxin administered at irregular time intervals of 12 to 18 months between each injection session.

An exclusion criterion was females who had previous operative procedures of any sort to their forehead, including endoscopic forehead lift. The study was conducted in private practice over a period of five years. Patients were followed up for $4-6$ months after the last injection session.

All patients were grade II or III (Carruther's) classification of forehead lines. Botulinum toxin type A "Botox" of Allergan vials of 100 units, were each diluted in 2.5 cc of $0.9 \%$ normal fresh saline preparation, and withdrawn in 40 units syringes; thus one syringe mark was equivalent to one unit of Botulinum toxin type A. A fixed dosage of 20 units of Botulinum toxin type A injected to treat forehead lines was standardized in all forty females Figure(1).

All female patients were instructed to regularly visit the doctor two weeks after the initial injection, and four to six months later, which is the effective duration of Botulinum toxin type A. The score of Carruther's classification was subjectively and objectively determined and compared between the two groups of females; all data concerning patients included in this study were recorded in their individual sheets as well as dates of Botulinum toxin A injection. A written consent of approval for this study was obtained as well as photographic data

\section{Figures(2-6).}

In the two groups, some females did need complementary methods in addition to Botulinum toxin A injection to reach more satisfactory and aesthetically pleasing results. The intervention included hyaluronic acid 
injections, facial resurfacing using Erbium Yag laser and fat injection.

Evaluation of the aesthetic outcome was done subjectively by patient self- evaluation with four Likert subscales for the degree of satisfaction (very satisfied, satisfied, less satisfied and unsatisfied). Objective evaluation was assessed through photographic data by three medical personnel not involved in the study, also using four Likert subscales (very good, good, fair and poor) Graph(2).

\section{Results:}

In group A, 17 females, (85\%) showed no change of their frontal forehead lines after five years of Botulinum toxin A treatment. Six of the eight patients (30\%) who started as grade II scale, remained at the same scale score after 5 years. Eleven of the twelve patients who were grade III scale also remained at the same grade. Two patients of group A score II, (10\%) showed changes from grade II to grade III Carruther's score during the five year period. One female, $(5 \%)$, with a score III changed to score IV after five years. Botulinum toxin type A proved effective and sufficient to the majority of females in this group. Two of the three females who showed one grade changes after five years asked for complementary Erbium Yag fractionated laser resurfacing in addition to the Botulinum injections, and one patient had hyaluronic acid injection to further enhance fine horizontal forehead lines, although she was satisfied with the overall result after five years. For all twenty females of this group, surgery was not an issue for discussion.

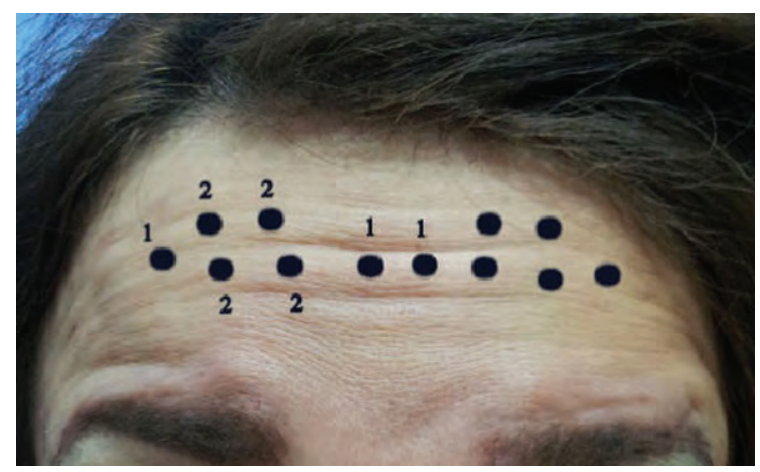

Figure (1):Forehead rhytides injected with a fixed dose of 20 units Botulinum Toxin A.
In group B, 16 of the 20 females, (80\%), showed changes from their original forehead scale score after five years. Three of the seven patients $(15 \%)$ who started with grade score II showed a change from grade II to grade IV scale. The other four patients (20\%) showed a change from grade II to grade III scale score, and nine $(45 \%)$ of the remaining thirteen patients showed a change from grade III to IV scale. Four females (20\%) with grade II score of this group showed no grade change within the five year period $\mathbf{G r a p h}(\mathbf{1} \mathbf{a} \& \mathbf{b})$.

Eleven females in this group seeked additional methods for horizontal forehead lines enhancement. Two females (16\%) did correctional fat injection to treat deep forehead creases, and six other females (30\%) had hyaluronic acid injections to the forehead creases two weeks after Botulinum toxin type A. Two patients performed Erbium Yag fractionated laser resurfacing treatment in addition to hyaluronic acid and Botulinum toxin injections. One female (5\%) discussed the possibility of a forehead lift procedure, but never did the operation to date.

A peculiar finding in both groups was noticed, which was the appearance of a horizontal line just above the lateral aspect of the eyebrow $(0.5-1 \mathrm{~cm})$ on one or both sides. The finding was seen in five patients $(25 \%)$ of group A, and nine females (45\%) in group B. As minimal as two units of the toxin were injected at the site for fear of brow ptosis, but the lines still showed little tendency to become less apparent by time.

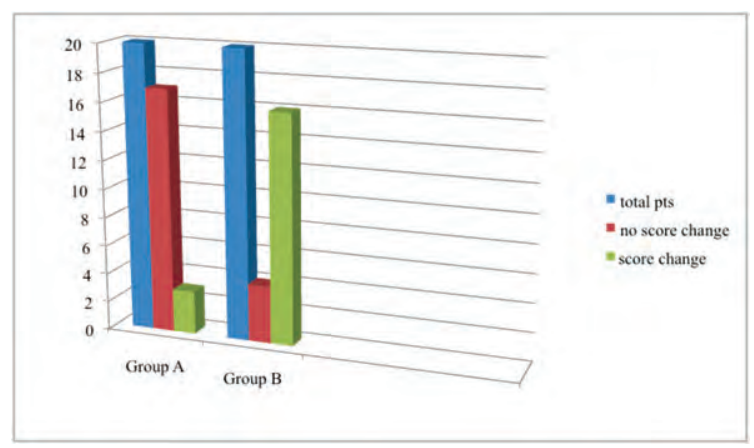

Graph (1a): Showing the total no. of patients, patients with score or no score changes according to Carruther's scale for forehead rhytides. 

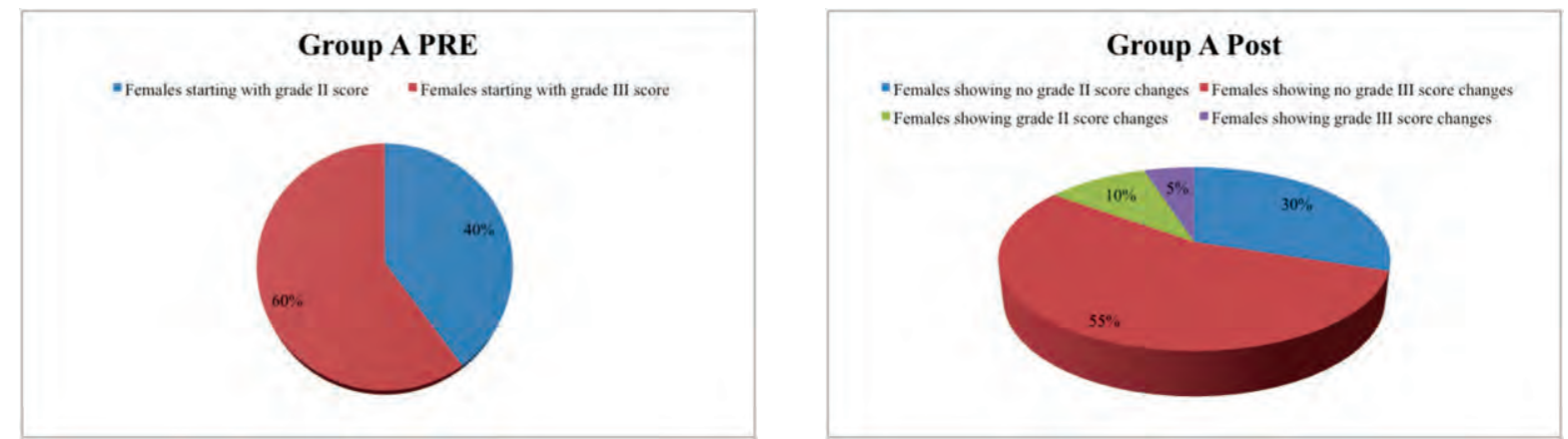

Group A
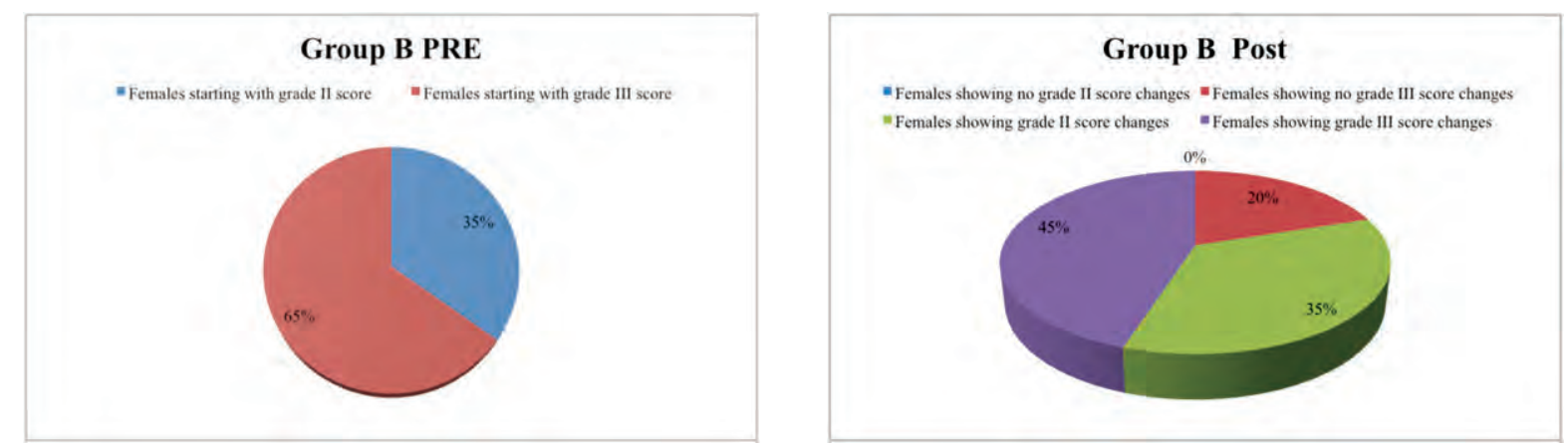

Group B

Graph (1b): showing percentage of patients with starting grade scores of horizontal forehea. lines and the percentage of females with forehead line changes after five years
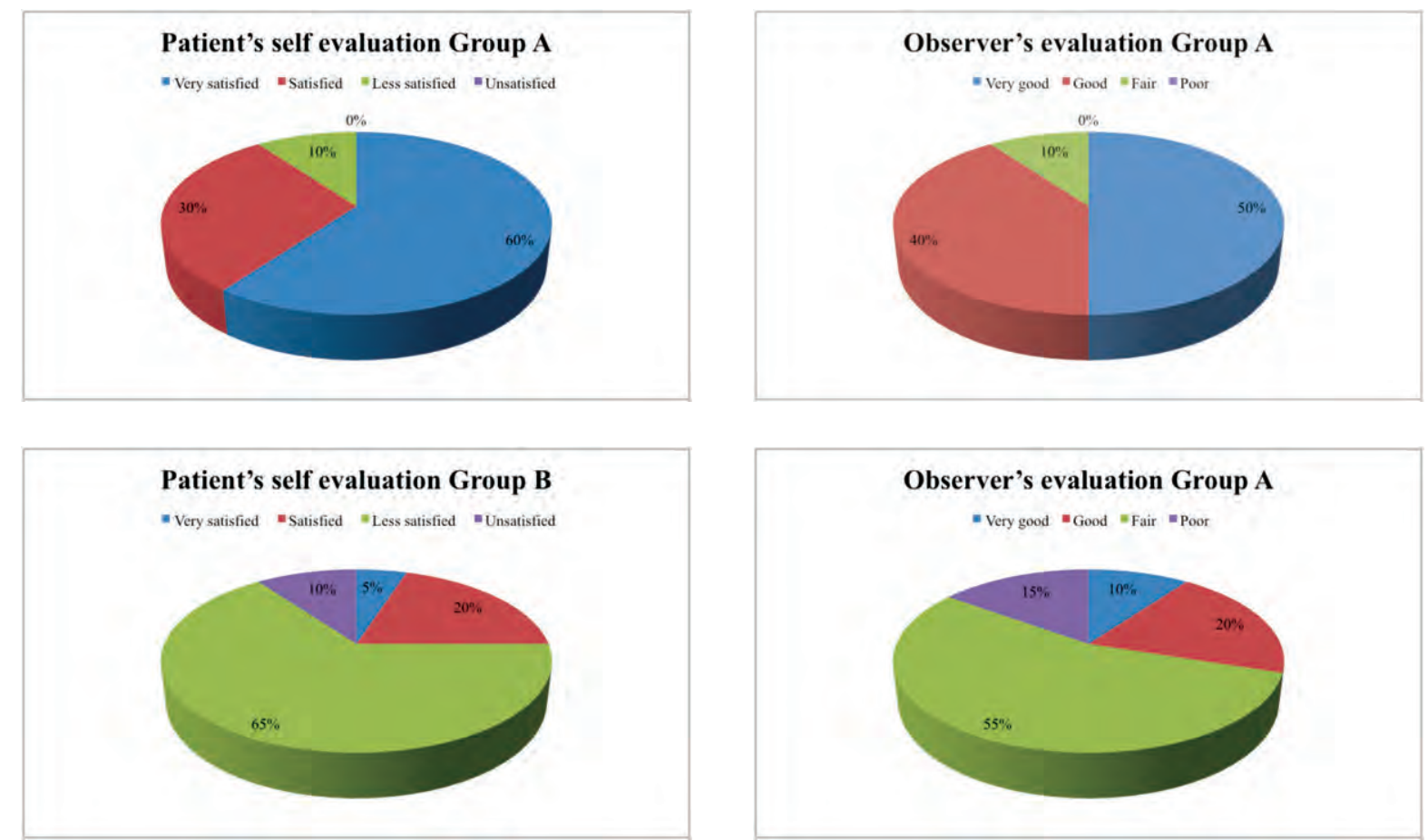

Graph (2):Showing Likert's objective and subjective evaluation of the five year study. 

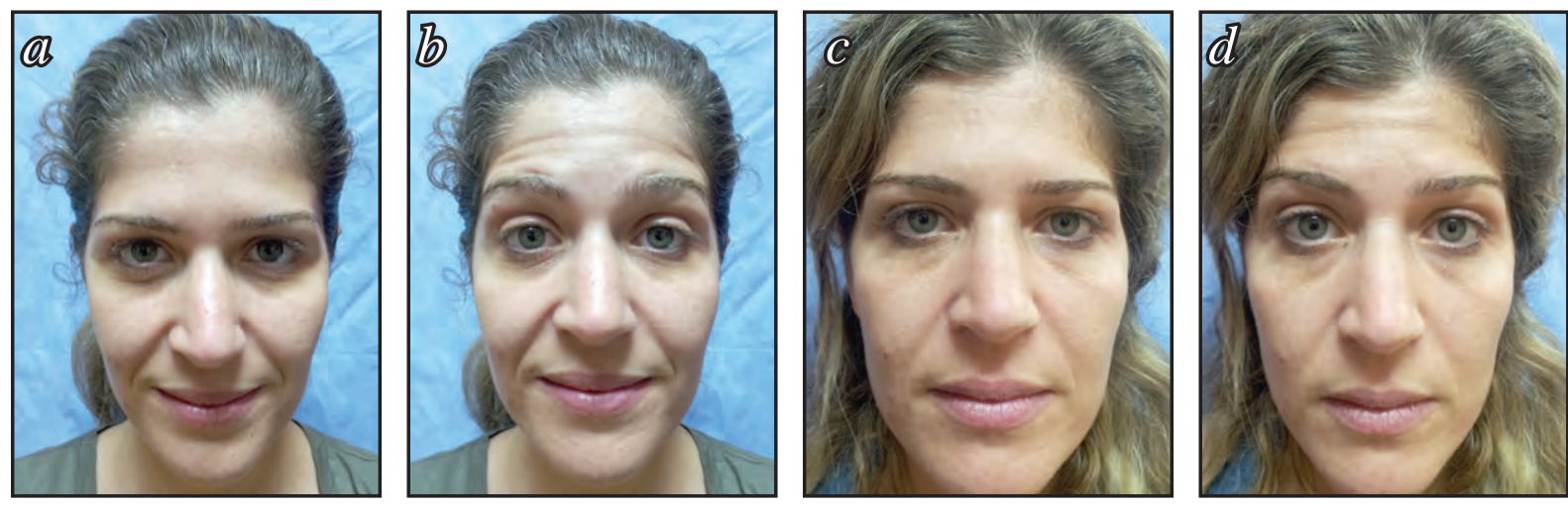

Figure (2a-d): $a \& b$ : patient at $1^{\text {st }}$ session, $c \& d: 5$ years later
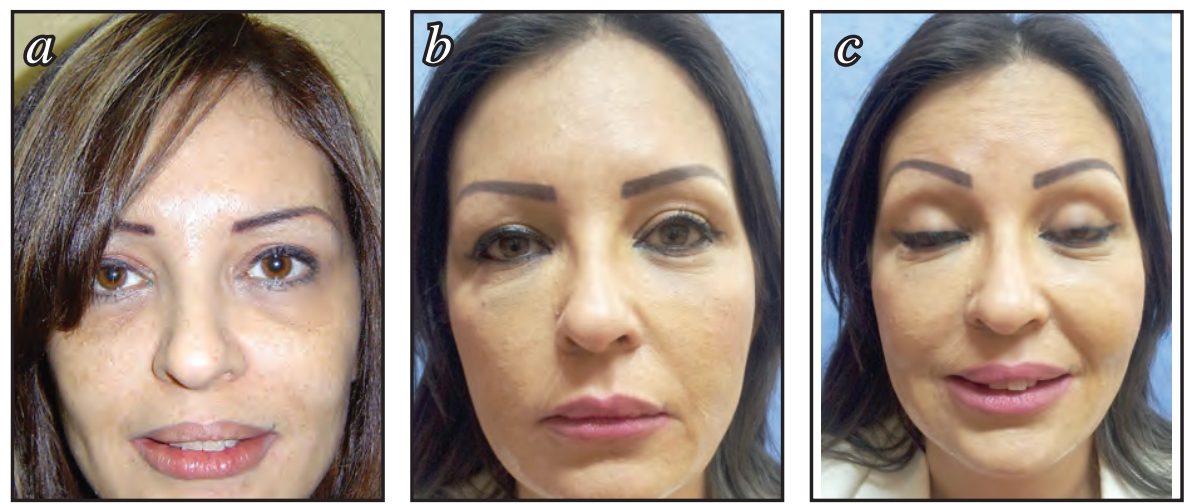

Figure (3a-c): a: patient at $1^{\text {st }}$ session, $b \& c: 5$ years later.
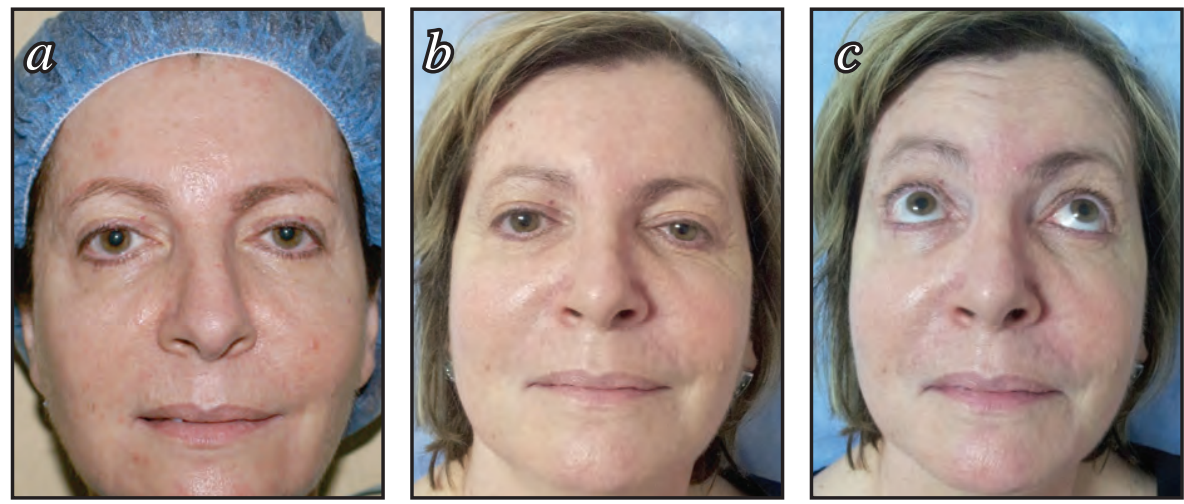

Figure (4a-c): a: patient at $1^{\text {st }}$ session, $b \& c: 5$ years later.

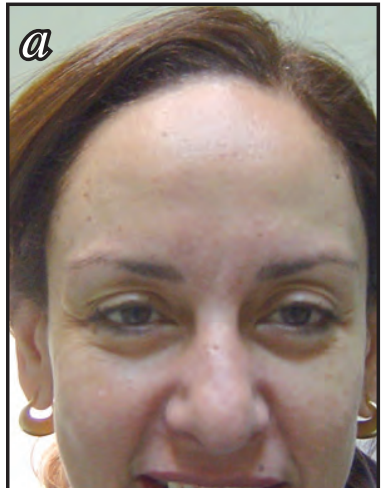

Figure (5a): $1^{\text {st }}$ session

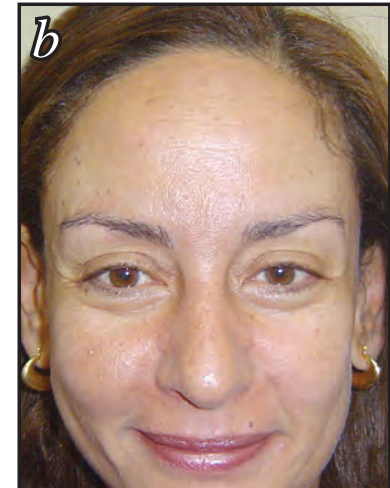

Figure (5b): 5 years later one grade change

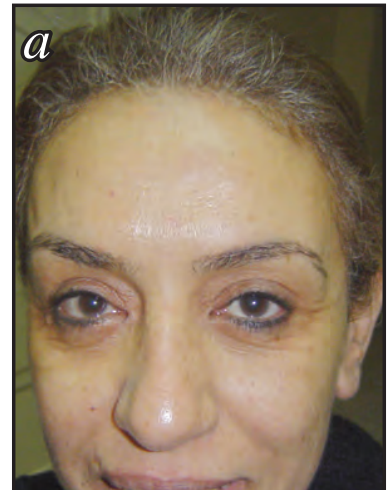

Figure (6a): $1^{\text {st }}$ session

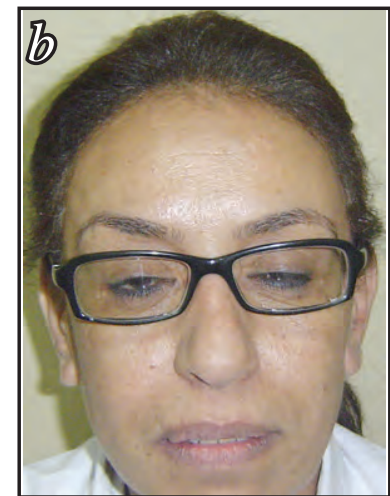

Figuer (6b): 5 years later one grade change 


\section{Discussion:}

With advancement of non-surgical cosmetic procedures, various choices are now available to both patients and doctors. Treating horizontal forehead wrinkles starts in a step ladder manner, beginning with Botulinum toxin type A injection alone or together with hyaluronic acid fillers. Furthermore, chemical peeling or laser resurfacing may also be added. Minimal surgery to the forehead including brow lift, and full forehead lift either endoscopically or surgically, are usually the patients' last choice, unless severe skin damage demands operative intervention from the start. ${ }^{9}$

Different classifications of forehead wrinkles were used in an attempt to standardize the management, but the two mostly accepted and used are the Glogau classification (1996), and the Carruther's modification grading (2008).

Kinetic frontal forehead wrinkles usually start to become bothersome to a considerable number of women by their mid-30's, and by the time they seek professional care for the first time, $56 \%$ of them have grade III forehead wrinkles. ${ }^{10}$ These findings coincide with the findings in our study, as 25 of the 40 women first presented with grade III score for forehead wrinkles. This represents $63 \%$ of cases.

The cumulative effects of external and internal factors will finally change the nature of facial rhytides to become deeper or more in number; the only concern is the rate of that change. Women may appear younger or older than their real age. Botulinum toxin type A injections may delay the need for surgical upper face rejuvenation later in life. ${ }^{11}$

According to photographic data collected over four years of the initial status and progress of forehead rhytides, the Carruthers established a validated photo-numeric scale for horizontal forehead rhytides in 2008 . Photographic data also verified that with scheduled administration of Botulinum toxin A, surgical intervention for upper face rejuvenation can be postponed. In a study of 60 female patients regularly scheduled for Botulinum toxin A injection over a period of four years, only $11 \%$ of them needed additional procedures besides the toxin injection.

In our study, similar findings were recorded for group A females regularly scheduled for the toxin injection over a five year period. $10 \%$ needed additional laser resurfacing to Botulinum injection, but the thought of surgery even in the near future, was not a demand for any patients in this group.

The Carruther's, back in 2007 commented on the eyebrow height after Botulinum toxin A injections, and explained the wrinkles above the lateral aspect of the eyebrow to be due to inactivation of the medial fibers of the frontalis muscle after treatment. This results in increased muscle fiber activity in the lateral and superior frontalis muscle fibers. ${ }^{12}$

On the other hand, Kang et al, 2011, described exaggeration of wrinkles after regular Botulinum toxin type A injection for forehead horizontal lines. They described the appearance of one new deep wrinkle just above the eyebrow in two cases. The authors explained this as being caused by hyperactivity and overcompensation of untreated muscles. ${ }^{13}$

In our study, the deep forehead line that would appear just above the eyebrow on one or both sides though, was a finding in five females $(25 \%)$ of group A who had regular toxin administration, and nine patients in group B $(45 \%)$. This area is usually either not treated with Botulinum toxin injection or minimally treated ( 2 units) due to fear of eyebrow ptosis. Our explanation of the exaggerated lateral supra eyebrow lines maybe due to the fact that mobility of the medial aspect of the eyebrow is limited compared to the lateral aspect. The explanation coincides with the study conducted by Sullivant et al 2006 on 12 cadavers. Anatomical dissection showed three medial retaining ligaments of the eyebrow as compared to one lateral retaining ligament. ${ }^{14}$

It was clear that females who had injections of Botulinum toxin type A administered at regular time intervals, showed superior results of forehead rejuvenation than those who had the toxin injected over longer periods of time. 


\section{Conclusion:}

Regular administration of Botulinum toxin type A within its effective period every 4 to 6 months, delays the worsening and deepening of frontal forehead rhytides. A healthy lifestyle, less exposure to the sun and no smoking all help delay deterioration of horizontal forehead lines. The need for surgical intervention thus to correct forehead rhytides may be postponed at a later stage of individuals life.

Infrequent Botulinum toxin type A injections, together with harmful external factors affecting and unhealthy lifestyle, give the impression of an older looking female compared to her real age and render the need for surgery at an earlier stage of life.

\section{References:}

1- Kaltreider SA, Kennedy RH, Woog JJ, Bradley EA, Custer PL, Meyer DR: American Academy of Ophthalmology; Ophthalmic Technology Assessment Committee Oculoplastics Panel. Cosmetic oculofacial applications of Botulinum toxin: A report by the American Academy of Ophthalmology. Ophthalmology 2005; 112(6): 1159-1167.

2- Flynn TC, Carruthers A, Carruthers J, Geister TL, Görtelmeyer R, Hardas B, Himmrich S, Kerscher M, de Maio M, Mohrmann C, Narins RS, Pooth R, Rzany B, Sattler G, Buchner L, Benter U, Fey C, Jones D: Validated assessment scales for the upper face. Dermatol Surg 2012; 38(2 Spec No.): 309-19.

3- Nestor MS, Ablon GR: The frontalis activity measurement standard: A novel contralateral method for assessing Botulinum neurotoxin type-A activity. $J$ Drugs Dermatol 2011; 10(9): 968-972.

4- Berbos ZJ, Lipham WJ: Update on Botulinum toxin and dermal fillers. Curr Opin Ophthalmol 2010; 21(5): 387-395.

5- Sternick I, Pitanguy I: Botulinum toxin dosage template for frontal wrinkle effacement. Aesthet Surg J 2011; 31(7): 814-820.
6- Binder WJ: Long-term effects of Botulinum toxin type A (Botox) on facial lines: Acomparison in identical twins. Arch Facial Plast Surg 2006; 8(6): 426-431.

7- Glogau RG: Aesthetic and anatomic analysis of the aging skin. Semin Cutan Med Surg 1996; 15(3): 134-138.

8- Carruthers A, Carruthers J, Hardas B, Kaur M, Goertelmeyer R, Jones D, Rzany B, Cohen J, Kerscher M, Flynn TC, Maas C, Sattler G, Gebauer A, Pooth R, McClure K, Simone-Korbel U, Buchner L: A validated grading scale for forehead lines. Dermatol Surg 2008; 34 (2): 155-160.

9- Coleman KR, Carruthers J: Combination therapy with BOTOX and fillers: The new rejuvnation paradigm. Dermatol Ther 2006; 19(3): 177-188.

10-Farahvash MR, Arad S: Clostridium botulinum type A toxin for the treatment of upper face animation lines: An Iranian experience. J Cosmet Dermatol 2007; 6(3): 152-158.

11-Abbasi NR, Durfee MA, Petrell K, Dover JS, Arndt KA: A small study of the relationship between Botulinum toxin A concentration and forehead wrinkle reduction. Arch Dermatol 2012; 148(1): 119-121.

12-Carruthers A, Carruthers J: Eyebrow height after Botulinum toxin type A to the glabella. Dermatol Surg 2007; 33 (1 spec No.): 26-31.

13-Kang SM, Feneran A, Kim JK, Park O, Kim JE, Won $\mathrm{CH}$, Chang S, Lee MW, Choi JH, Moon KC, Youn CS, Cho S, Lee SH: Exaggeration of wrinkles after botulinum toxin injection for forehead horizontal lines. Ann Dermatol 2011; 23(2): 217-221.

14-Sullivan PK, Salomon JA, Woo AS, Freeman MB: The importance of the retaining ligamentous attachments of the forhead for selective eyebrow reshaping and forehead rejuvenation. Plast Reconstr Surg 2006; 117 (1): 95-104. 